\title{
Phase I Dose-Finding Study of OPB-111077, a Novel STAT3 Inhibitor, in Patients with Advanced Hepatocellular Carcinoma
}

\author{
Changhoon Yoo, MD 1 \\ Jihoon Kang, MD, \\ Ho Yeong Lim, MD, PhD2 \\ Jee Hyun Kim, MD, PhD ${ }^{3}$ \\ Myung-Ah Lee, MD, PhD ${ }^{4}$ \\ Kyung-Hun Lee, MD, PhD 5 \\ Tae-You Kim, MD, PhD \\ Baek-Yeol Ryoo, MD, PhD 1
}

\begin{abstract}
${ }^{1}$ Department of Oncology, Asan Medical Center, University of Ulsan College of Medicine, Seoul, ${ }^{2}$ Division of Hematology-Oncology, Department of Medicine, Samsung Medical Center, Sungkyunkwan University School of Medicine, Seoul, ${ }^{3}$ Department of Internal Medicine, Seoul National University Bundang Hospital, Seoul National University College of Medicine, Seongnam, ${ }^{4}$ Division of Medical Oncology, Department of Internal Medicine, Seoul St. Mary's Hospital, College of Medicine, The Catholic University of Korea, Seoul, ${ }^{5}$ Department of Internal Medicine, Seoul National University Hospital, Seoul National University College of Medicine, Seoul, Korea
\end{abstract}

Correspondence: Baek-Yeol Ryoo, MD, PhD Department of Oncology, Asan Medical Center, University of Ulsan College of Medicine, 88 Olympic-ro 43-gil, Songpa-gu,

Seoul 05505, Korea

Tel: 82-2-3010-3211

Fax: 82-2-3010-6961

E-mail: ryooby@amc.seoul.kr

Received April 18, 2018

Accepted June 12, 2018

Published Online June 13, 2018

*Changhoon Yoo and Jihoon Kang contributed equally to this work.

aPresent address: Division of Hematology / Oncology, Department of Internal Medicine, Kangbuk Samsung Hospital, Sungkyunkwan University School of Medicine, Seoul, Korea

\section{Purpose}

The signal transducer and activator of transcription 3 (STAT3) signaling pathway might be a promising therapeutic target for hepatocellular carcinoma (HCC).

\section{Materials and Methods}

This study was a multicenter, open-label, non-comparative, dose escalating phase I study of OPB-111077, an oral STAT3 inhibitor, in patients with advanced HCC who failed on sorafenib. Continuous dosing (daily administration, 50 to $400 \mathrm{mg}$ ) and intermittent dosing (4-days on/3-days off administration: 300 to $900 \mathrm{mg}$ ) regimens were evaluated and the dose-limiting toxicities (DLTs), maximum tolerated dose (MTD), and recommended dose (RD) were the primary endpoints.

\section{Results}

A total of 33 patients (19 for continuous dosing and 14 for intermittent dosing) were enrolled. One patient experienced a DLT with grade 3 dizziness, but the MTD was identified in neither the continuous nor the intermittent dosing cohorts. The RDs were determined to be $250 \mathrm{mg}$ for the continuous dosing regimen and $600 \mathrm{mg}$ for the intermittent dosing regimen. There was no treatment-related death; five patients (15.2\%) had grade 3-4 toxicities including thrombocytopenia (6\%), fatigue (3\%), and dizziness (3\%). No patients achieved complete or partial responses and the median progression-free survival was 1.4 months (95\% confidence interval, 0.8 to 2.8 ).

\section{Conclusion}

OPB-111077 was well tolerated in patients with advanced HCC after sorafenib failure, but only showed limited preliminary efficacy outcomes. Further investigation of the role of the STAT3 signaling pathway in HCC and the development of biomarkers for STAT3 inhibitors are warranted. 


\section{Introduction}

Hepatocellular carcinoma (HCC) is the most common type of liver cancer. In the last decade, it has become one of the most frequently occurring tumors worldwide, and it is the third highest cause of cancer-related death globally [1]. In Korea, HCC remains one of most common causes of cancerrelated mortality, although the incidence rate of liver cancer has decreased since 1999 [2].

For patients with early-stage localized disease, surgical resection, liver transplantation, and ablative therapy are potentially curative treatment options [3]. Chemoembolization is the mainstay of treatment for patients with HCC confined to the liver with preserved liver function [3]. However, patients with HCC diagnosed at the advanced stages, or which has progressed after locoregional therapy, show poor prognosis. Sorafenib, a multikinase inhibitor, has been established as a standard first-line systemic chemotherapy for patients with advanced HCC based on the success of pivotal phase III trials [4,5]. After 10 years of failures in the development of systemic treatment for HCC, regorafenib, an oral multikinase inhibitor, recently demonstrated improved survival in patients who progressed on sorafenib in the randomized phase III RESORCE trial [6], and nivolumab, an antiprogrammed-cell-death protein 1 (PD-1) inhibitor, showed promising activity in a non-comparative phase I/II CheckMate 040 trial [7]. Despite these recent advances, the survival of patients with advanced HCC remains dismal and novel approaches to target and inhibit hepatocarcinogenesis are required to improve survival outcomes.

Signal transducer and activator of transcription 3 (STAT3) belongs to the STAT family of proteins, which have functions as both signal transducers and transcription factors [8]. The aberration of STAT3 appears to be inherent in its relationships with multiple types of hematological and solid cancers [8]. A link between STAT3 and HCC has been previously suggested, as enhanced production of interleukin 6 and tumor necrosis factor through the activation of STAT3 is critical for obesity-promoted HCC preclinical models [9,10], and the STAT3 signaling pathway is involved in multiple models of hepatocellular oncogenesis [11-13]. In addition, STAT3 inhibitors showed significant anti-cancer activity in preclinical HCC models [14]. This suggests that STAT3 is a promising target for the treatment of advanced HCC $[15,16]$. Although several STAT3 inhibitors were tested in advanced solid cancers, including HCC, no agent has been proven to be effective in a randomized trial [17-19].

OPB-111077 is an orally bioavailable STAT3 inhibitor which has shown preclinical efficacy in both in vitro and in vivo preclinical HCC models. Based on these rationales, we conducted a multicenter, open-label, dose-finding phase I study of OPB-111077 for patients with advanced HCC.

\section{Materials and Methods}

This study was a multicenter, open-label, non-comparative, dose escalating phase I study in patients with advanced HCC, to determine the dose-limiting toxicities (DLTs), maximal tolerated dose (MTD), and recommended dose (RD) of OPB-111077. The dosing escalation scheme included a continuous dosing (daily administration) and intermittent dosing (4-days on/3-days off administration) regimen. This study was conducted at five academic institutions in Korea.

\section{Patients}

Patients with HCC, confirmed by a pathological or noninvasive assessment according to the American Association for the Study of Liver Diseases criteria for patients with confirmed cirrhosis, were eligible for inclusion in this study if they met the following criteria: age between 20 and 75 years; Eastern Cooperative Oncology Group (ECOG) performance status of $0-1$; extrahepatic metastasis or locally advanced disease not amenable to surgical resection or loco-regional therapies; Child-Pugh classification A or B (except 9); documented evidence of unresponsiveness to, intolerance to, or ineligibility for sorafenib; and estimated life expectancy of 12 weeks or greater.

\section{Study design and treatment}

A standard, multiple dose, 3+3 study design was used, and consisted of two dosing regimen cohorts: a continuous dosing regimen for once daily administration (5 cohorts with doses of $50 \mathrm{mg}, 100 \mathrm{mg}, 150 \mathrm{mg}$, $200 \mathrm{mg}$, and $250 \mathrm{mg}$, respectively) and an intermittent dosing regimen for 4-days on/ 3-days off administration (3 cohorts with doses of $300 \mathrm{mg}$, $500 \mathrm{mg}$, and $600 \mathrm{mg}$, respectively). Both dosing regimens consisted of a 24-day initial treatment cycle (cycle 1), and 21day subsequent treatment cycles (cycle 2 onwards) (Fig. 1).

This study was originally designed to evaluate the continuous dosing regimen; however, intermittent dosing cohort was added subsequently based on the findings of a prior phase I study of OPB-111077 for patients with unselected advanced cancers [20] that all DLTs occurred at the 300 $\mathrm{mg} /$ day and $400 \mathrm{mg} /$ day dose levels at approximately 1 week after the start of administration. Setting a washout period (3 days) was considered to ease the management of the enhancement of toxicity in the daily regimen of OPB111077. 


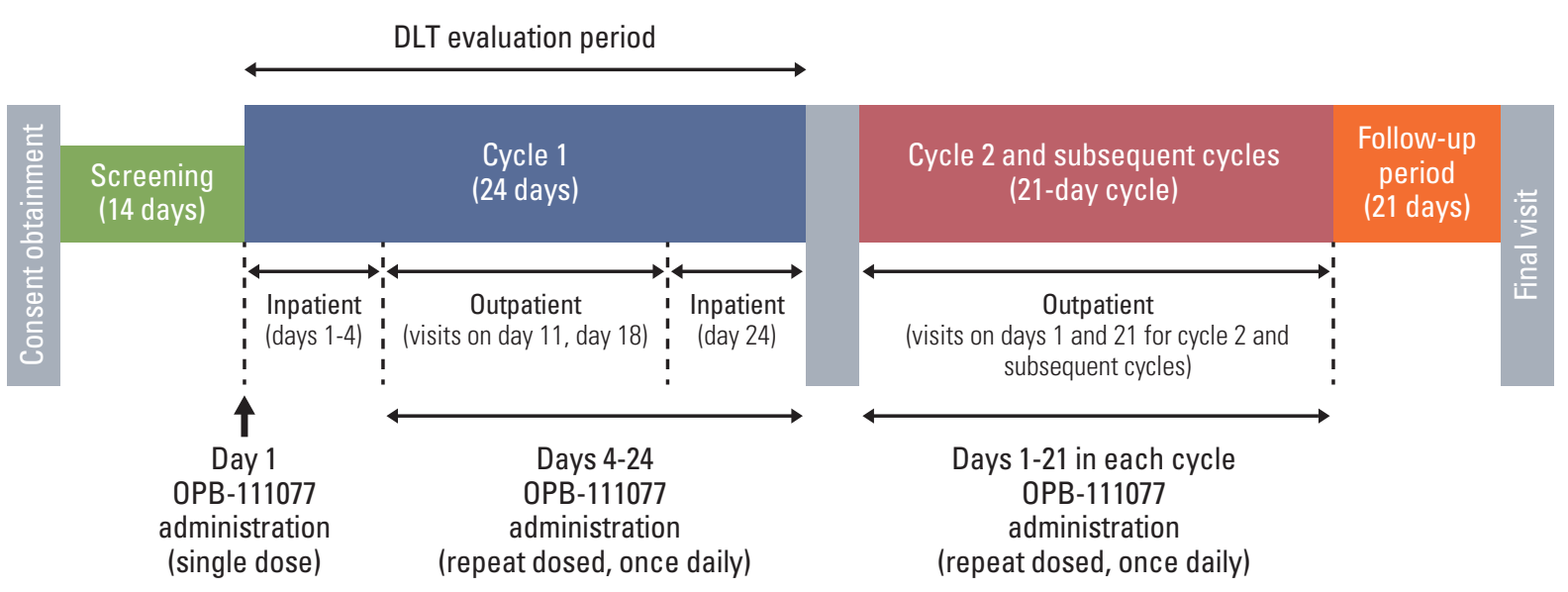

B

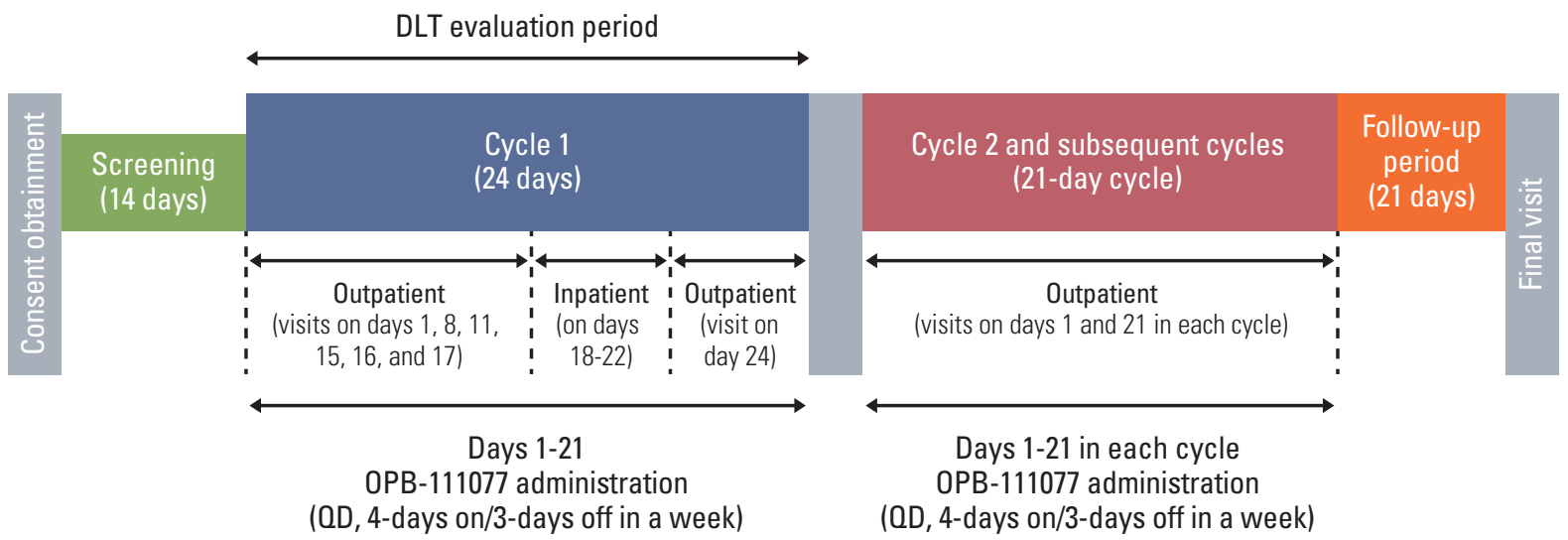

Fig. 1. Study design. Dose escalation in the continuous dosage schedule with once daily administration (A) and the intermittent dosage schedule with 4-days on/3-days off administration (B).

OPB-111077 was administered as a single oral dose at approximately the same time each morning. In cycle 1 of the continuous dosing regimen, patients received OPB-111077 on day 1 , followed by a 2-day treatment-free interval for safety and pharmacokinetic (PK) evaluation. OPB-111077 administration resumed on day 4 and continued through day 24. In the intermittent dosing regimen cohort, patients received OPB-111077 for 4 days each week for 3 weeks (day 1 to 21), followed by a 3-day treatment-free period (days 22 to 24). Study treatment continued until patients experienced intolerable toxicity, the occurrence of disease progression, or withdrawal of consent. Cycle 2 and subsequent cycles consisted of 3 weeks (21 days) with no treatment-free intervals.

Doses were reduced or interrupted according to a prespecified protocol if grade 2 or greater non-hematological toxicities or grade 3 or greater hematological toxicities occurred. Study treatment was discontinued if there was dose interruption of more than 21 days despite appropriate supportive care. Dose reduction was allowed up to two levels and the lowest dose was $25 \mathrm{mg}$ in the continuous dosing regimen or $200 \mathrm{mg}$ in the intermittent dosing regimen.

\section{Evaluation}

Safety profiles were evaluated by adverse events (AEs), vital sign, body weight, electrocardiogram, laboratory tests, ECOG performance status, and physical examinations. AEs were assessed and graded according to the National Cancer Institute Common Terminology Criteria for Adverse Events (NCI-CTCAE), ver. 4.0. 
Efficacy outcomes were measured using radiological assessments, including computed tomography/magnetic resonance imaging scans, and graded according to the Response Evaluation Criteria In Solid Tumors ver. 1.1. The same method of assessment and the same techniques were used to characterize each identified and reported lesion at the baseline and follow-up stages.

\section{Dose-limiting toxicities}

A DLT is defined as an AE with a causal relationship with the study treatment occurring in cycle 1 (Supplementary Methods). Unless they experienced a DLT, patients with drug compliance of $<75 \%$ during cycle 1 were excluded from the DLT evaluation and additional patients were enrolled.

\section{PK analysis}

Plasma was obtained for the determination of concentrations of OPB-111077 and its metabolites at days 1-4, 11, 18, and 24 in the continuous regimen cohort, and days $1,8,15$, 16,17 , and 18 in the intermittent regimen cohort. Details of the pharmacokinetic analyses are summarized in the supplementary methods.

\section{Statistical analysis}

The primary endpoint was to determine the DLTs, MTD, $\mathrm{RD}$, and safety profiles of OPB-111077 as a continuous or intermittent dose. The secondary endpoints include the PK parameters of OPB-111077, overall response rate, disease control rate (DCR) at 6 weeks and 12 weeks, progression-free survival (PFS), and overall survival (OS). The full analysis set included patients who received at least one dose of OPB111077 and from whom post-treatment efficacy data were available. The safety analysis set consisted of all subjects that received at least one dose of OPB-111077.

\section{Ethical statement}

The study protocol was approved by the institutional review boards of participating centers, and all patients provided written informed consent before enrolment. The study was conducted in accordance with the Declaration of Helsinki and the Guidelines for Good Clinical Practice (ClinicalTrial.gov Identifier: NCT01942083).
Table 1. Baseline patient characteristics

\begin{tabular}{lc} 
Characteristic & Value $(\mathbf{n}=33)$ \\
Dosing cohort & \\
Continuous dosing & $19(57.6)$ \\
Intermittent dosing & $14(42.4)$ \\
Age (yr) & $54(23-69)$ \\
Male sex & $27(81.8)$ \\
Etiology & \\
\hline HBV infection & $26(78.8)$ \\
\hline Alcohol & $5(15.1)$ \\
\hline Others & $2(6.1)$ \\
\hline ECOG performance status & \\
\hline 0 & $10(30.3)$ \\
1 & $23(69.7)$ \\
Extrahepatic metastasis & $33(100)$ \\
\hline Lung & $27(81.8)$ \\
Lymph nodes & $12(36.4)$ \\
\hline Peritoneum & $6(18.2)$ \\
Bone & $5(15.2)$ \\
Previous sorafenib & $33(100)$ \\
Previous lines of systemic treatment & $1(1-7)$ \\
1 & $23(69.7)$ \\
2 & $2(6.1)$ \\
3 & $6(18.2)$ \\
\hline 5 & $1(3.0)$ \\
7 & $1(3.0)$ \\
\hline
\end{tabular}

Values are presented as number (\%) or median (range). HBV, hepatitis B virus; ECOG, Eastern Cooperative Oncology Group.

\section{Results}

\section{Patients}

A total of 33 patients (19 for the continuous dosing cohort and 14 for the intermittent dosing cohort) were enrolled. Their baseline patient characteristics are summarized in Table 1. Cycle 1 of the study treatment was completed in 27 patients $(81.8 \%$ ) and DLTs were evaluable in 30 patients. All patients were Korean and had ECOG performance status of $0-1$. Median age was 54 years (range, 23 to 69 years) and most patients $(81.8 \%)$ were male. Hepatitis B virus infection was the most common etiology of HCC $(n=26,78.8 \%)$. Except for three patients $(9.1 \%)$ with Child-Pugh B, all patients were classified as Child-Pugh A at baseline. All patients had extrahepatic metastasis and the most common metastatic sites were the lung $(\mathrm{n}=27,82 \%)$ and lymph nodes $(\mathrm{n}=12$, $36 \%$ ). All patients previously received sorafenib and median 1 (range, 1 to 7 ) systemic chemotherapy regimen, including 
Table 2. Adverse events occurring in $>10 \%$ of patients

\begin{tabular}{|c|c|c|c|c|}
\hline Adverse event & $\begin{array}{l}\text { All patients } \\
\quad(\mathrm{n}=33)\end{array}$ & $\begin{array}{l}\text { Continuous dosing } \\
\text { cohort }(n=19)\end{array}$ & $\begin{array}{l}\text { Intermittent dosing } \\
\text { cohort }(n=14)\end{array}$ & p-value \\
\hline Any grade TEAE & $33(100)$ & $19(100)$ & $14(100)$ & $>0.99$ \\
\hline Grade 3-4 TEAE & $5(15.2)$ & $2(10.5)$ & $3(21.4)$ & 0.63 \\
\hline Abdominal pain & $5(15.2)$ & $3(15.8)$ & $2(14.3)$ & $>0.99$ \\
\hline Grade 3-4 & $1(3.0)$ & $1(5.3)$ & 0 & $>0.99$ \\
\hline Constipation & $6(18.2)$ & $2(10.5)$ & $4(28.6)$ & 0.36 \\
\hline Grade 3-4 & 0 & 0 & 0 & - \\
\hline Cough & $4(12.1)$ & $3(15.8)$ & $1(7.1)$ & 0.62 \\
\hline Grade 3-4 & 0 & 0 & 0 & - \\
\hline Decreased appetite & $12(36.4)$ & $10(52.6)$ & $2(14.3)$ & 0.02 \\
\hline Grade 3-4 & 0 & 0 & 0 & - \\
\hline Diarrhea & $4(12.1)$ & $1(5.3)$ & $3(21.4)$ & 0.29 \\
\hline Grade 3-4 & 0 & 0 & 0 & - \\
\hline Dizziness & $14(42.4)$ & $5(26.3)$ & $9(64.3)$ & 0.03 \\
\hline Grade 3-4 & $1(3.0)$ & $1(5.3)$ & 0 & $>0.99$ \\
\hline Fatigue & $14(42.4)$ & $10(52.6)$ & $4(28.6)$ & 0.17 \\
\hline Grade 3-4 & $1(3.0)$ & 0 & $1(7.1)$ & 0.42 \\
\hline Hemoptysis & $4(12.1)$ & $4(21.1)$ & 0 & 0.12 \\
\hline Grade 3-4 & 0 & 0 & 0 & - \\
\hline Headache & $9(27.3)$ & $7(36.8)$ & $2(14.3)$ & 0.24 \\
\hline Grade 3-4 & 0 & 0 & 0 & - \\
\hline Hypothyroidism & $4(12.1)$ & $2(10.5)$ & $2(14.3)$ & $>0.99$ \\
\hline Grade 3-4 & 0 & 0 & 0 & - \\
\hline Myalgia & $4(12.1)$ & $2(10.5)$ & $2(14.3)$ & $>0.99$ \\
\hline Grade 3-4 & 0 & 0 & 0 & - \\
\hline Nausea & $28(84.6)$ & $17(89.5)$ & $11(78.6)$ & 0.63 \\
\hline Grade 3-4 & 0 & 0 & 0 & - \\
\hline Thrombocytopenia & $4(12.1)$ & $1(5.3)$ & $3(21.4)$ & 0.29 \\
\hline Grade 3-4 & $2(6.1)$ & 0 & $2(14.3)$ & 0.17 \\
\hline Pyrexia & $4(12.1)$ & $1(5.3)$ & $3(21.4)$ & 0.29 \\
\hline Grade 3-4 & 0 & 0 & 0 & - \\
\hline Vomiting & $16(48.5)$ & $6(31.6)$ & $10(71.4)$ & 0.02 \\
\hline Grade 3-4 & 0 & 0 & 0 & - \\
\hline
\end{tabular}

Values are presented as number (\%). TEAE, treatment emergent adverse event.

sorafenib, was administered prior to study enrollment.

\section{Dose limiting toxicities}

One patient in the $250 \mathrm{mg}$ continuous dosing cohort experienced a DLT of grade 3 dizziness. DLTs were not observed in the intermittent dosing cohort. The MTD was not identified in either the continuous or intermittent dosing cohorts. However, considering the PK profiles and increased frequencies of AEs with dose escalation, the RDs were determined to be $250 \mathrm{mg}$ for continuous dosing and $600 \mathrm{mg}$ for intermittent dosing.

\section{Adverse events}

All 33 patients were included in the safety analysis (Table 2). The duration of study treatment was median 22 days (range, 4 to 209 days); 40 days (range, 4 to 209 days) in the continuous dosing cohort and 12 days (range, 7 to 59) in the intermittent dosing cohort. There was no treatment-related death. All patients had at least one treatment-emergent AE (TEAE) and 5 patients $(15.2 \%)$ had grade $3-4$ TEAEs $(10.5 \%$ in the continuous dosing cohort and $21.4 \%$ in the intermittent dosing cohort). The most frequently reported TEAEs were nausea $(84.6 \%)$ and vomiting $(48.5 \%)$. Although there was no dose-related trend in the frequency of TEAEs, there was an 


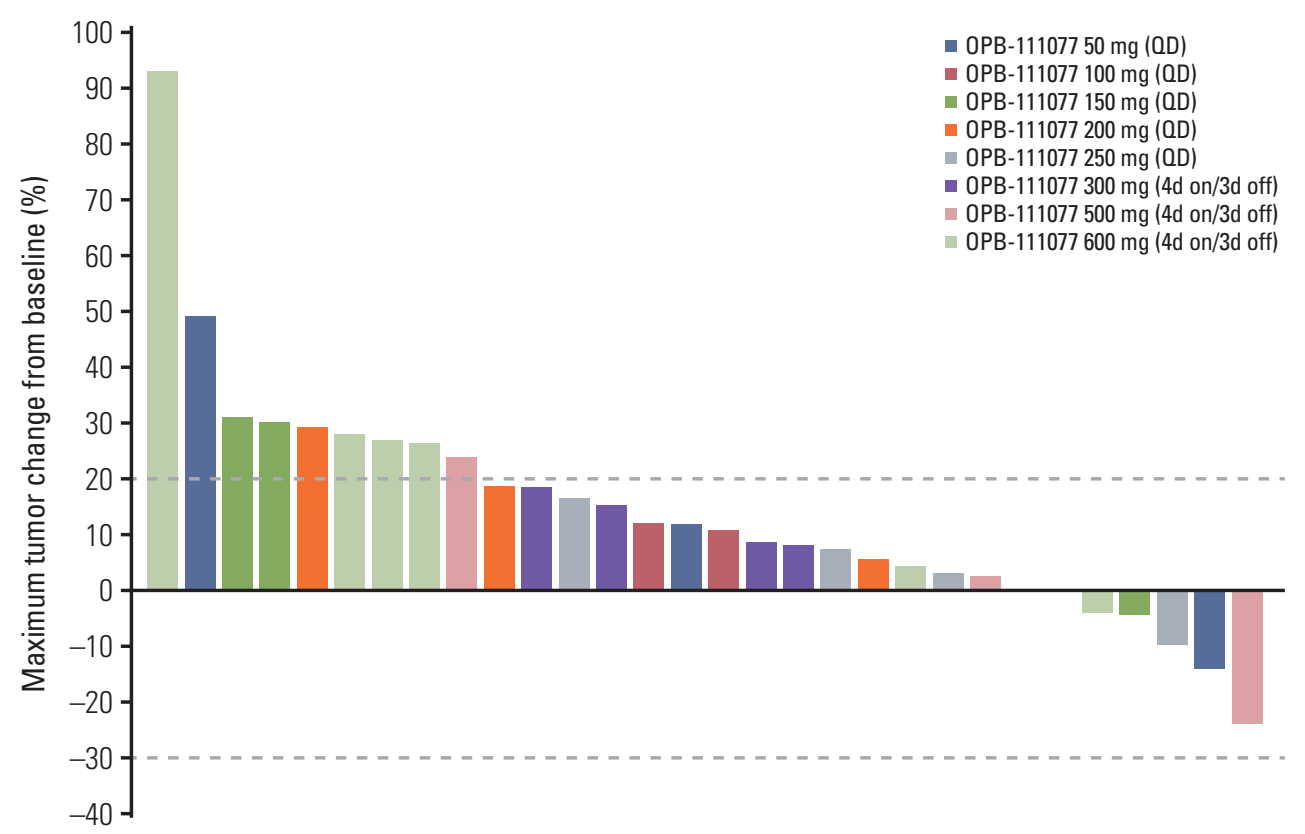

Fig. 2. Waterfall plot of the changes in the target lesion. $4 \mathrm{~d}$ on/3d off, 4-days on/3-days off administration.

increase in the severity of nausea in the highest dose level (600 mg, 4-days on/3-days off), where most patients (6 of 7, $85.7 \%$ ) experienced grade 2 nausea. The most frequent grade 3-4 TEAEs was thrombocytopenia $(n=2,6.1 \%)$. In the intermittent dosing cohort, vomiting ( $71.4 \%$ vs. $31.6 \%, \mathrm{p}=0.02)$ and dizziness ( $64.3 \%$ vs. $26.3 \%, \mathrm{p}=0.03$ ) were significantly more common but decreased appetite was less common $(14.3 \%$ vs. $52.6 \%)$ than in the continuous dosing cohort.

TEAEs led to treatment discontinuation in three patients due to grade 3 abdominal pain and dizziness (both in the 200 $\mathrm{mg}$ continuous dosing cohort) and grade 4 thrombocytopenia (in the $300 \mathrm{mg}$ intermittent dosing cohort). Dose reduction and interruption occurred in three ( 1 in the continuous and 2 in the intermittent dosing cohort) and five patients ( 1 in the continuous and 4 in the intermittent dosing cohort), respectively.

\section{Efficacy}

A total of 31 patients were included in the efficacy assessment. There were no patients who achieved complete or partial responses. Stable disease and progressive disease were the best responses in $13(42 \%)$ and $18(58 \%)$ patients, respectively. Five of 30 patients $(16.7 \%)$ with measurable lesions showed reductions in the sizes of target lesions (Fig. 2). There was no significant difference in the rate of stable disease between dosing regimen $(44.4 \%$ in the continuous dosing cohort vs. $38.5 \%$ in the intermittent dosing cohort; $\mathrm{p}>0.99$ ).

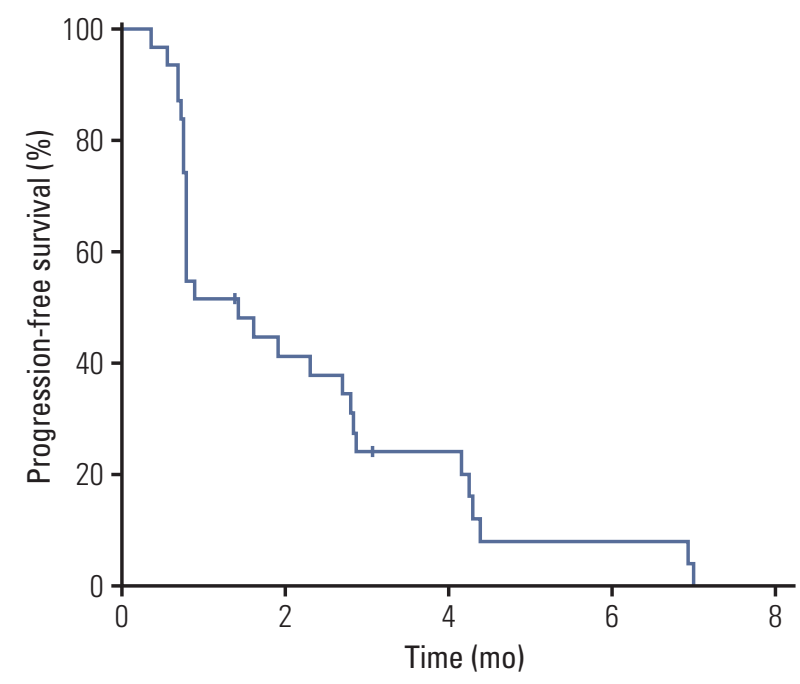

Fig. 3. Kaplan-Meier estimates of progress-free survival in overall patients. The median value was 1.4 months $(95 \%$ confidence interval 0.8 to 2.8 ).

The DCRs at 6 weeks and 12 weeks were $41.9 \%$ and $22.6 \%$, respectively. There were also no dose-related trends in tumor response in either dosing regimen (S1 Table).

Median PFS was 1.4 months (95\% confidence interval [CI], 0.8 to 2.8 ) and there was no significant difference between dosing regimens (median 1.6 months [95\% CI, 0.8 to 4.2 ] in 
the continuous dosing cohort vs. 0.9 months [ $95 \%$ CI, 0.7 to $2.8]$ in the intermittent dosing cohort; $\mathrm{p}=0.21$ ) (Fig. 3). Median OS was not reached at the time of analysis and 6-month and 1-year OS rates were $44.4 \%$ and $11.1 \%$, respectively.

\section{PK parameters}

The results of PK analyses of the continuous dosing regimen and intermittent dosing regimen cohorts are listed in S2-S4 Tables. OPB-111077 was readily absorbed after both single and multiple doses, with median $t_{\max }$ values ranging from 1 to 4.96 hours across dose levels and dosing regimens. The half-life $\left(t_{1 / 2}\right)$, following multiple dose administration, ranged from 12.7 to 15.5 hours and was comparable across dosing levels and dosing regimens.

\section{Discussion}

This study was the phase 1 trial of the novel STAT3 inhibitor, OPB-111077, for patients with advanced HCC after failure of sorafenib. Two dosing schemes, continuous daily dosing and intermittent dosing (4-days on /3-days off administration), were tested to determine the DLTs, MTD, and RD. The safety profile, $\mathrm{PK}$, and preliminary efficacy of each dosing schedule was also evaluated.

In this trial, the MTD was not determined in either the continuous or intermittent dosing cohorts. One patient experienced grade 3 dizziness, a prespecified DLT, in the $250 \mathrm{mg}$ continuous dosing cohort, while no patients showed DLTs in the intermittent dosing cohorts. Considering the PK profiles and the increased frequency of AEs, particularly gastrointestinal symptoms, with dose escalation, the RDs of OPB-111077 for further evaluation in HCC populations were determined as $250 \mathrm{mg}$ once daily for continuous dosing, and $600 \mathrm{mg}$ once daily, 4-days on/3-days off, for intermittent dosing. These were consistent with the results of a previous phase I trial of OPB-111077 performed in United States, which included various types of cancers [20]. In this trial, the MTD of continuous daily OPB-111077 dosing was $250 \mathrm{mg}$ once daily, and the observed DLTs were grade 3 nausea/ vomiting at a dose of $400 \mathrm{mg}$ once daily and grade 3 dizziness at a dose of $300 \mathrm{mg}$ once daily.

PK analysis showed that OPB-111077 was readily absorbed after both single and multiple doses in both the continuous and intermittent dosing cohorts. Because of the small sample sizes, the data in this study were insufficient to reliably assess dose proportionality with inferential statistics. However, our results were in line with those of previous studies performed in United States [20].
OPB-111077 was well tolerated and showed favorable safety profiles. Most TEAEs were of grade 1 or 2 and manageable with appropriate supportive care. Nausea and vomiting were the most frequent TEAEs, and occurred in $84.6 \%$ and $48.5 \%$ of patients, respectively. Vomiting and dizziness were more commonly observed in the intermittent dosing cohorts, and decreased appetite in the continuous dosing cohorts. Grade 3-4 TEAEs occurred in $15.2 \%$ of patients and thrombocytopenia $(6.1 \%)$, dizziness $(3.0 \%)$, and fatigue $(3.0 \%)$ were the most common severe toxicities. These safety profiles of OPB-111077 in HCC patients are consistent with the results of the previous phase 1 study of OPB-111077 in which continuous dosing for various cancer types showed that nausea $(68.5 \%)$, vomiting $(48 \%)$, fatigue $(59.8 \%)$, and dizziness $(31.5 \%)$ were common toxicities [20]. Previous trials that investigated other STAT3 inhibitors also reported that gastrointestinal symptoms, including nausea and vomiting, are the major TEAEs, with generally favorable safety profiles $[17,18]$.

Despite $16.7 \%$ of patients with measurable lesions showing reduction in the sizes of target lesions, no patients achieved objective responses in this study, and the median PFS was only 1.4 months. There was no difference in efficacy between the different dosing schedules. Although based on a small number of patients, the current preliminary efficacy outcome did not justify the further evaluation of OPB-111077 in advanced HCC, considering that other investigational agents tested in randomized trials at the second-line setting after the failure of sorafenib showed median PFS (or time-to-progression) and OS of at least 3 months and 8 months, respectively $[6,7,21-23]$. Regorafenib, which has been approved as secondline therapy for sorafenib-progressed advanced HCC, showed a median PFS and OS of 3.1 months and 10.6 months, respectively [6]. An anti-PD-1 inhibitor, nivolumab, showed promising results in phase I/II trial with objective response rates of $15 \%$ and a median PFS of 4 months [7].

Despite the strong rationale for the investigation of STAT3 inhibition in HCC $[9,11,14,24]$, the efficacy of small molecule STAT3 inhibitors, including ours, were limited [19]. In a previous Japanese study investigating another STAT3 inhibitor, OPB-31121, no patients showed objective responses and the median PFS was approximately 2 months in patients with HCC [19]. These findings indicate that STAT3 inhibitors, as a monotherapy, might not be effective in patients with HCC, and there are several hypotheses to explain these failures. First, there is the possibility that STAT3 inhibition was insufficient with these agents to produce anti-cancer activity. Because pharmacodynamic evaluation such as the measurement of STAT3 phosphorylation in peripheral blood was not done in these studies, it is not clear whether STAT3 was effectively targeted. Second, there is the lack of biomarkers for STAT3 inhibitors. Janus kinases (JAKs) are the upstream 
regulators of the JAK-STAT3 signaling pathway and ruxolitinib, a JAK2 inhibitor, is effective for patients with myelofibrosis and polycythemia vera in which JAK2 mutations are prevalent [25]. In contrast, there is no proven biomarker for STAT3 inhibitors and this limits the ability to stratify the patients who would benefit most from STAT3 inhibitors in the trial. Recent advances in understanding the role of STAT3 signaling in the development of HCC might help to revisit STAT3 inhibitors in subsets of HCC patients, such as those with FGF19-driven HCC or obesity-related HCC $[9,12]$.

Although OPB-111077 showed limited efficacy as a monotherapy in patients with HCC, this agent might be further investigated as a combination therapy, considering its favorable safety profiles. Because the STAT3 signaling pathway has an immunomodulatory role in cancer development [26], the combination of OPB-111077 with anti-PD-1 inhibitors might be synergistic. The efficacy of OPB-111077 in combination with currently approved agents including sorafenib or regorafenib, or with promising investigational agents including FGFR inhibitors, might be also valuable to investigate.

In conclusion, OPB-111077 was well tolerated in patients with advanced HCC after failure of sorafenib, but only showed limited preliminary efficacy outcomes. Further investigation of the role of the STAT3 signaling pathway in HCC and the development of biomarkers for STAT3 inhibitors are warranted.

\section{Electronic Supplementary Material}

Supplementary materials are available at Cancer Research and Treatment website (https: // www.e-crt.org).

\section{Conflicts of Interest}

This study was funded by Otsuka Pharmaceutical.

\section{References}

1. El-Serag HB. Hepatocellular carcinoma. N Engl J Med. 2011; 365:1118-27.

2. Jung KW, Won YJ, Oh CM, Kong HJ, Lee DH, Lee KH, et al. Cancer statistics in Korea: incidence, mortality, survival, and prevalence in 2014. Cancer Res Treat. 2017;49:292-305.

3. Bruix J, Sherman M; American Association for the Study of Liver D. Management of hepatocellular carcinoma: an update. Hepatology. 2011;53:1020-2.

4. Llovet JM, Ricci S, Mazzaferro V, Hilgard P, Gane E, Blanc JF, et al. Sorafenib in advanced hepatocellular carcinoma. N Engl J Med. 2008;359:378-90.

5. Cheng AL, Kang YK, Chen Z, Tsao CJ, Qin S, Kim JS, et al. Efficacy and safety of sorafenib in patients in the Asia-Pacific region with advanced hepatocellular carcinoma: a phase III randomised, double-blind, placebo-controlled trial. Lancet Oncol. 2009;10:25-34.

6. Bruix J, Qin S, Merle P, Granito A, Huang YH, Bodoky G, et al. Regorafenib for patients with hepatocellular carcinoma who progressed on sorafenib treatment (RESORCE): a randomised, double-blind, placebo-controlled, phase 3 trial. Lancet. 2017;389:56-66.

7. El-Khoueiry AB, Sangro B, Yau T, Crocenzi TS, Kudo M, Hsu $C$, et al. Nivolumab in patients with advanced hepatocellular carcinoma (CheckMate 040): an open-label, non-comparative, phase 1/ 2 dose escalation and expansion trial. Lancet. 2017; 389:2492-502.

8. Al Zaid Siddiquee K, Turkson J. STAT3 as a target for inducing apoptosis in solid and hematological tumors. Cell Res. 2008; 18:254-67.

9. Park EJ, Lee JH, Yu GY, He G, Ali SR, Holzer RG, et al. Dietary and genetic obesity promote liver inflammation and tumorigenesis by enhancing IL-6 and TNF expression. Cell. 2010;140: 197-208.

10. Toffanin S, Friedman SL, Llovet JM. Obesity, inflammatory signaling, and hepatocellular carcinoma-an enlarging link. Cancer Cell. 2010;17:115-7.

11. Hatziapostolou M, Polytarchou C, Aggelidou E, Drakaki A, Poultsides GA, Jaeger SA, et al. An HNF4alpha-miRNA inflammatory feedback circuit regulates hepatocellular oncogenesis. Cell. 2011;147:1233-47.

12. Zhou M, Yang H, Learned RM, Tian H, Ling L. Non-cellautonomous activation of IL-6/STAT3 signaling mediates FGF19-driven hepatocarcinogenesis. Nat Commun. 2017;8: 15433.

13. Yan Q, Jiang L, Liu M, Yu D, Zhang Y, Li Y, et al. ANGPTL1 interacts with integrin alpha1beta1 to suppress HCC angiogenesis and metastasis by inhibiting JAK2/STAT3 signaling. Cancer Res. 2017;77:5831-45.

14. Lin L, Amin R, Gallicano GI, Glasgow E, Jogunoori W, Jessup JM, et al. The STAT3 inhibitor NSC 74859 is effective in hepatocellular cancers with disrupted TGF-beta signaling. Oncogene. 2009;28:961-72.

15. Llovet JM, Villanueva A, Lachenmayer A, Finn RS. Advances in targeted therapies for hepatocellular carcinoma in the genomic era. Nat Rev Clin Oncol. 2015;12:408-24.

16. Yu H, Lee H, Herrmann A, Buettner R, Jove R. Revisiting STAT3 signalling in cancer: new and unexpected biological functions. Nat Rev Cancer. 2014;14:736-46.

17. Wong AL, Soo RA, Tan DS, Lee SC, Lim JS, Marban PC, et al. Phase I and biomarker study of OPB-51602, a novel signal 
transducer and activator of transcription (STAT) 3 inhibitor, in patients with refractory solid malignancies. Ann Oncol. 2015;26:998-1005.

18. Oh DY, Lee SH, Han SW, Kim MJ, Kim TM, Kim TY, et al. Phase I study of OPB-31121, an oral STAT3 inhibitor, in patients with advanced solid tumors. Cancer Res Treat. 2015; $47: 607-15$.

19. Okusaka T, Ueno H, Ikeda M, Mitsunaga S, Ozaka M, Ishii H, et al. Phase 1 and pharmacological trial of OPB-31121, a signal transducer and activator of transcription-3 inhibitor, in patients with advanced hepatocellular carcinoma. Hepatol Res. 2015;45:1283-91.

20. Tolcher A, Flaherty K, Shapiro GI, Berlin J, Witzig T, Habermann T, et al. A first-in-human phase I study of OPB-111077, a small-molecule STAT3 and oxidative phosphorylation inhibitor, in patients with advanced cancers. Oncologist. 2018; 23:658-e72.

21. Zhu AX, Park JO, Ryoo BY, Yen CJ, Poon R, Pastorelli D, et al. Ramucirumab versus placebo as second-line treatment in patients with advanced hepatocellular carcinoma following first-line therapy with sorafenib (REACH): a randomised, double-blind, multicentre, phase 3 trial. Lancet Oncol. 2015;16:85970.
22. Zhu AX, Kudo M, Assenat E, Cattan S, Kang YK, Lim HY, et al. Effect of everolimus on survival in advanced hepatocellular carcinoma after failure of sorafenib: the EVOLVE-1 randomized clinical trial. JAMA. 2014;312:57-67.

23. Llovet JM, Decaens T, Raoul JL, Boucher E, Kudo M, Chang $\mathrm{C}$, et al. Brivanib in patients with advanced hepatocellular carcinoma who were intolerant to sorafenib or for whom sorafenib failed: results from the randomized phase III BRISKPS study. J Clin Oncol. 2013;31:3509-16.

24. Li WC, Ye SL, Sun RX, Liu YK, Tang ZY, Kim Y, et al. Inhibition of growth and metastasis of human hepatocellular carcinoma by antisense oligonucleotide targeting signal transducer and activator of transcription 3. Clin Cancer Res. 2006;12: 7140-8.

25. Deisseroth A, Kaminskas E, Grillo J, Chen W, Saber H, Lu HL, et al. U.S. Food and Drug Administration approval: ruxolitinib for the treatment of patients with intermediate and high-risk myelofibrosis. Clin Cancer Res. 2012;18:3212-7.

26. Zhang M, Liu Q, Mi S, Liang X, Zhang Z, Su X, et al. Both miR17-5p and miR-20a alleviate suppressive potential of myeloidderived suppressor cells by modulating STAT3 expression. J Immunol. 2011;186:4716-24. 